Full Length Article

\title{
Association Mapping of Grain Weight, Length and Width in Barley (Hordeum vulgare) Breeding Germplasm
}

\author{
Yunping Lai ${ }^{1,2 \dagger}$, Y. Yu ${ }^{2 \dagger}$, X. Liu' ${ }^{1}$ H. Wan' ${ }^{2}$ Z. Zhang ${ }^{2}$, L. Wang ${ }^{2}$, Y. Leng ${ }^{2}$ L. Ma ${ }^{1,3}$, W. Yang ${ }^{2}$ and Z. Feng ${ }^{*}$ \\ ${ }^{1}$ College of Agronomy, Sichuan Agricultural University, Chengdu 611130, Sichuan, China \\ ${ }^{2}$ Crop Research Institute, Sichuan Academy of Agricultural Sciences, Chengdu 610066, Sichuan, China \\ ${ }^{3}$ College of Environmental Sciences, Sichuan Agricultural University, Chengdu 611130, Sichuan, China \\ *For correspondence: zyfeng49@126.com \\ †These authors contributed equally to this paper
}

\begin{abstract}
Grain weight, grain length and grain width are important target traits in the malting barley breeding, due to their attributes for determining the market value of barley grain. In this study, a diversity collection of 112 accessions was used to identify quantitative trait loci (QTLs) for them by association mapping approach with simple sequence repeat (SSR) markers and detect elite alleles related to the improvement of grain size. The heritability calculated for grain weight over multi-environment experiments was $83.6 \%$, while the values of grain length and width were $70.0 \%$ and $69.9 \%$, respectively. Population structure analysis showed that all the accessions were divided into two subgroups that originated from East Asia and North America. A total of five QTLs for grain weight were identified on chromosomes $1 \mathrm{H}, 2 \mathrm{H}, 5 \mathrm{H}$ and $6 \mathrm{H}$, respectively, and the QTLs on 5HS and 6HS were detected in more than one environment. The A4 allele of GBM1215 on 6HS increased grain weight significantly in all three environments. Four and two QTLs were detected for grain length and width, respectively. QTL for grain width on $2 \mathrm{HS}$ was detected in two environments and the $A 1$ allele produced wider grain. These findings might be valuable for marker-assisted selection of breeding lines to improve grain weight and width. C) 2017 Friends Science Publishers
\end{abstract}

Keywords: Barley (Hordeum vulgare L.); Association mapping; Grain weight; Grain length; Grain width

\section{Introduction}

Barley (Hordeum vulgare L.) is the fourth most important cereal crop in the world and its grain is mainly used for animal feed, malting and brewing (Virender and Zhang, 2003; Walker et al., 2013). As one of the most adaptable cereals, the growing zone of barley ranges from subarctic to subtropical areas (Wenzel et al., 2015). Tibetan Plateau and its vicinity are considered as one of the centers of genetic diversity and domestication of cultivated barley (Feng et al., 2006a; Feng et al., 2006b; Dai et al., 2012). A larger number of wild barleys have been found in Tibet, Qinghai and Sichuan province of China, with high genetic diversity (Feng et al., 2006b; Dai et al., 2012). It was reported that Chinese cultivated barley originated from the tworowed wild barley from Tibet was significantly different from those found by other centers of genetic diversity (Dai et al., 2012).

Grain size including grain weight, grain length and grain width are important attributes for determining the market value of barley grain. Heavy and plump grains are always associated with superior malting quality, owing to their highsu malt extract, and better feed quality with more starch per grain (Hadjichristodoulou, 1990). Grain weight is also one component of yield (Hadjichristodoulou, 1990; Passarella et al., 2005), which is mainly determined by grain length and grain width (Sun et al., 2013; Rasheed et al., 2014). Thus, enhancing grain size is an efficient approach to improve both end-use quality and yield potential of barley.

Grain weight, length and width are complex quantitative traits, which are controlled by polygenes or QTLs (Sun et al., 2013) and influenced by environment (Xing and Zhang, 2010; Walker et al., 2013), i.e. temperature during pre-anthesis and grain filling (Wallwork et al., 1998; Passarella et al., 2005), drought stress (Royo et al., 2000), etc. In the past few decades, with the help of molecular markers and marker-based genetic maps, identifications of QTLs for grain weight in barley have been reported in different barley germplasms. According to those reports, QTLs for grain weight are almost distributed on all the seven chromosomes of barley (Kjaer and Jensen, 1996; Tinker et al., 1996; Bezant et al., 1997; Marquezcedillo et al., 2001; Li et al., 2005; Szücs et al., 2009; Worch et al.,

To cite this paper: Lai, Y., Y. Yu, X. Liu, H. Wan, Z. Zhang, L. Wang, Y. Leng, L. Ma, W. Yang and Z. Feng, 2017. Association mapping of grain weight, length and width in barley (Hordeum vulgare) breeding germplasm. Int. J. Agric. Biol., 19: 1175-1186 
2011; Walker et al., 2013). Matthies et al. (2012) detected several QTLs for grain weight on chromosomes $1 \mathrm{H}, 3 \mathrm{H}$ and $7 \mathrm{H}$ by association mapping using simple sequence repeat (SSR) and Diversity Arrays Technology (DArT) markers, most of which were consistent with QTLs detected in reported bi-parental populations. On chromosome $2 \mathrm{H}$, the intervals of several reported QTLs for grain weight were overlapped in different germplasms (Bezant et al., 1997; Marquezcedillo et al., 2001; Szücs et al., 2009; Mikolajczak et al., 2016; Wang et al., 2016). Moreover, major QTLs for grain weight on chromosome 4H (Pillen et al., 2003; Korff et al., 2006; Maurer et al., 2016) were also identified around the vernalization gene ' $\mathrm{Vrn}-\mathrm{H2}$ ' (Yan et al., 2004). QTLs for grain length and grain width were detected throughout most chromosomes (Backes et al., 1995; Ayoub et al., 2002; Walker et al., 2013). The putative candidate gene of one major grain length QTL on chromosome $4 \mathrm{H}$ that explained $22.3 \%$ of the phenotypic variance was homologous to $A n-1$ in rice, which encodes a bHLH protein regulating cell division, grain length, and awn elongation (Zhou et al., 2016).

Linkage mapping based on bi-parental cross population and association mapping (AM) are considered as two main approaches in QTLs detection. AM method that had been firstly applied in human genetics for complex diseases was based on linkage disequilibrium (LD) in diversity collection of germplasms. In plant, the population for AM covers larger number of alleles used in breeding, and takes most historical recombination events into account (Flint-Garcia et al., 2003; Gupta et al., 2005; Breseghello and Sorrells, 2006a), with advantages of increased involved genetic variations and resolution for QTL detection. Currently, AM has been widely applied in identifying phenotype-markers associated in barley (Long et al., 2013; Zhou and Steffenson, 2013; Ziems et al., 2014).

Although lots of QTLs for grain weight and size related traits have been reported in recent years, comparison of effects between different QTL alleles from different germplasms were reported rarely, which made it difficult for breeders to select suitable parental lines. Moreover, prevailing artificial plant breeding in China adopts only a small number of main lines that had unfortunately narrowed the genetic base of modern cultivated barley and made a great bottleneck on genetic enhancement for yield. It seems that introducing extra germplasms and further dissecting elite alleles become an effective remedy.

In this study, a diversity association mapping panel of 112 barley accessions from China, Mexico and USA was evaluated over three environments using simple sequence repeat (SSR) markers with a mixed linear model approaches (MLM), and the effects for enhancing grain weight, length and width were further compared between different QTL alleles at detected sites by association mapping. The goals of this study were to identify loci associated with grain weight, length and width, and detect the elite allelic variations enhancing grain size.

\section{Materials and Methods}

\section{Plant Materials}

The diversity barley panel contained 112 accessions, including elite parental lines, advanced breeding materials, current cultivars and landraces with the origins of Gansu (China), Qinghai (China), Sichuan (China), Tibet (China), Mexico and USA, which were provided by the Barley Research Centre of Sichuan Agriculture University (Table 1). The whole genotypes consisted of 104 six-rowed and 8 two-rowed accessions. Among the collection, 77 are hull-less barleys and 35 are hulled barleys. Of the 104 six rowed barely lines, 73 are naked barleys and the others are hulled barleys.

\section{Trait Evaluation}

Field trials were conducted in the northwest of the Chengdu basin, Sichuan Province, China (103 $88^{\prime}$ E, $30^{\circ} 82^{\prime} \mathrm{N}$; $548 \mathrm{~m}$ above sea level) in the years of 2014 (CD2014) and 2015 (CD2015) and the hilly area of Nanchong, Sichuan Province, China (105 $97^{\circ}$ E, 30 $30^{\circ} 78^{\prime}$ $\mathrm{N} ; 680 \mathrm{~m}$ above sea level) in 2015 (NC2015). Both Chengdu and Nanchong are under a humid subtropical monsoon climate with the average annual rainfall from $887.3 \mathrm{~mm}$ to $927.6 \mathrm{~mm}$. Each field experiment was performed in a randomized complete block design with two replicates. Each plot of the field experiment had five rows with $1.5 \mathrm{~m}$ long and spaced $0.35 \mathrm{~m}$ apart. After harvest, the grain weight was measured as the mean weight of 1000 individual grains (TGW), while grain length (GL) and grain width (GW) was defined as the longest and the widest distance through an average of 100 grains, respectively. All of three traits were conducted by the SC-G plant grain analysis system (Wseen company, China).

\section{Genotyping}

A total of 319 SSR markers, which were selected according to the consensus genetic map by Varshney et al. (2007) were used to genotyping. Each chromosome contained about 45 SSR markers for a balanceable coverage of barley chromosome. Genomic DNA was isolated from bulked young leaf tissue of 10 seedlings per accession using CTAB procedure (Irfan et al., 2013). PCR reactions were performed in a total volume of $15 \mu \mathrm{L}$ containing $1 \mathrm{x}$ Buffer, $2 \mathrm{mmol} \mathrm{L}^{-1} \mathrm{MgCl}_{2}, 0.25 \mathrm{mmol} \mathrm{L}^{-1} \mathrm{dNTPs}, 0.25 \mu \mathrm{mol} \mathrm{L}^{-1}$ of each primer, $1 \mathrm{U} r$ Taq-polymerase and $20 \mathrm{ng}$ genomic DNA as template. The PCR profile was as follows: one cycle of $94^{\circ} \mathrm{C}$ for $5 \mathrm{~min} ; 35$ cycles of $94^{\circ} \mathrm{C}$ for $45 \mathrm{sec}, 55-60^{\circ} \mathrm{C}$ (primer depended) for $45 \mathrm{~s}$ and $72^{\circ} \mathrm{C}$ for $1 \mathrm{~min}$; and a final extension at $72^{\circ} \mathrm{C}$ for $10 \mathrm{~min}$. The PCR products were separated in $6 \%(\mathrm{w} / \mathrm{v})$ denaturing polyacrylamide gels with $1 \mathrm{x}$ TBE Buffer and then were visualized by silver staining. 
Association Mapping of Grain Size in Barley / Int. J. Agric. Biol., Vol. 19, No. 5, 2017

Table 1: The code, name, origin and row type of 112 barley accessions

\begin{tabular}{|c|c|c|c|}
\hline Code & Name & Origin & Row type \\
\hline 1 & GQ2 & Gansu (China) & six \\
\hline 2 & GQ3 & Gansu & $\operatorname{six}$ \\
\hline 3 & GQ4 & Gansu & $\operatorname{six}$ \\
\hline 4 & 9516 & Gansu & $\operatorname{six}$ \\
\hline 5 & DLH & Gansu & $\operatorname{six}$ \\
\hline 6 & HZBQK & Gansu & $\operatorname{six}$ \\
\hline 7 & KJZBQK & Gansu & $\operatorname{six}$ \\
\hline 8 & MQK & Gansu & $\operatorname{six}$ \\
\hline 9 & HQK & Gansu & $\operatorname{six}$ \\
\hline 10 & $\mathrm{CQK}$ & Gansu & $\operatorname{six}$ \\
\hline 11 & HQK & Gansu & $\operatorname{six}$ \\
\hline 12 & SYQK & Gansu & $\operatorname{six}$ \\
\hline 13 & CQK & Gansu & $\operatorname{six}$ \\
\hline 14 & DMQK & Gansu & $\operatorname{six}$ \\
\hline 15 & LLGQK & Gansu & $\operatorname{six}$ \\
\hline 16 & LLLQK & Gansu & $\operatorname{six}$ \\
\hline 17 & XLQK & Gansu & $\operatorname{six}$ \\
\hline 18 & QLZQK & Gansu & $\operatorname{six}$ \\
\hline 19 & BQ1 & Qinghai (China) & $\operatorname{six}$ \\
\hline 20 & BQ2 & Qinghai & $\operatorname{six}$ \\
\hline 21 & BQ3 & Qinghai & $\operatorname{six}$ \\
\hline 22 & BQ5 & Qinghai & $\operatorname{six}$ \\
\hline 23 & BQ6 & Qinghai & $\operatorname{six}$ \\
\hline 24 & BQ7 & Qinghai & $\operatorname{six}$ \\
\hline 25 & KL2 & Qinghai & six \\
\hline 26 & KL10 & Qinghai & two \\
\hline 27 & KL12 & Qinghai & $\operatorname{six}$ \\
\hline 28 & HYTQK & Qinghai & $\operatorname{six}$ \\
\hline 29 & LDQK & Qinghai & $\operatorname{six}$ \\
\hline 30 & HZSCR & Qinghai & $\operatorname{six}$ \\
\hline 31 & DMDGLLQK & Qinghai & $\operatorname{six}$ \\
\hline 32 & HZBLLQK & Qinghai & $\operatorname{six}$ \\
\hline 33 & HZLLQK & Qinghai & six \\
\hline 34 & HYLLL & Qinghai & $\operatorname{six}$ \\
\hline 35 & DTBLS & Qinghai & $\operatorname{six}$ \\
\hline 36 & $\mathrm{CMBQK}$ & Qinghai & $\operatorname{six}$ \\
\hline 37 & LDBQK & Qinghai & $\operatorname{six}$ \\
\hline 38 & M112BQK & Qinghai & $\operatorname{six}$ \\
\hline 39 & YSBQK & Qinghai & $\operatorname{six}$ \\
\hline 40 & HUBLLQK & Qinghai & $\operatorname{six}$ \\
\hline 41 & DTBLL & Qinghai & $\operatorname{six}$ \\
\hline 42 & DTHJN & Qinghai & $\operatorname{six}$ \\
\hline 43 & HZHCM & Qinghai & $\operatorname{six}$ \\
\hline 44 & HZCM & Qinghai & $\operatorname{six}$ \\
\hline 45 & HZLCM & Qinghai & $\operatorname{six}$ \\
\hline 46 & YHCMQK & Qinghai & $\operatorname{six}$ \\
\hline 47 & XHXQK & Qinghai & $\operatorname{six}$ \\
\hline 48 & XHHQK & Qinghai & $\operatorname{six}$ \\
\hline 49 & HLWLQK & Qinghai & $\operatorname{six}$ \\
\hline 50 & MYQLQK & Qinghai & $\operatorname{six}$ \\
\hline 51 & ZYDM & Qinghai & $\operatorname{six}$ \\
\hline 52 & HYHQK & Qinghai & $\operatorname{six}$ \\
\hline 53 & REGBXHK & Qinghai & $\operatorname{six}$ \\
\hline 54 & GHHQK & Qinghai & $\operatorname{six}$ \\
\hline 55 & LDLQK & Qinghai & $\operatorname{six}$ \\
\hline 56 & HZZPQK & Qinghai & $\operatorname{six}$ \\
\hline 57 & MZQK & Qinghai & $\operatorname{six}$ \\
\hline 58 & FB0310 & Qinghai & $\operatorname{six}$ \\
\hline 59 & FBO226 & Sichuan (China) & $\operatorname{six}$ \\
\hline 60 & SPZQK & Sichuan & $\operatorname{six}$ \\
\hline 61 & QNQK & Sichuan & $\operatorname{six}$ \\
\hline 62 & BYQK & Sichuan & $\operatorname{six}$ \\
\hline 63 & AQ4 & Sichuan & six \\
\hline 64 & AQ5 & Sichuan & $\operatorname{six}$ \\
\hline 65 & BDCQK & Tibet (China) & $\operatorname{six}$ \\
\hline
\end{tabular}

Table 1: Continued

\begin{tabular}{|c|c|c|c|}
\hline 66 & BDQK & Tibet & six \\
\hline 67 & FB0641 & Tibet & six \\
\hline 68 & FB0642 & Tibet & six \\
\hline 69 & FB0647 & Tibet & six \\
\hline 70 & FB0648 & Tibet & six \\
\hline 71 & FB0650 & Tibet & six \\
\hline 72 & FB0651 & Tibet & six \\
\hline 73 & Golas Bley & Mexico & two \\
\hline 74 & B3034 & Mexico & two \\
\hline 75 & Tibetannia & Mexico & two \\
\hline 76 & K5 & Mexico & two \\
\hline 77 & ARUMIR & USA & $\operatorname{six}$ \\
\hline 78 & WAS3 & USA & two \\
\hline 79 & APM-HB1905 & USA & six \\
\hline 80 & H.SAT.V.HAX.F.FURB & USA & six \\
\hline 81 & $\mathrm{SAH}$ & USA & six \\
\hline 82 & AHOR443170 & USA & six \\
\hline 83 & AHOR2194170 & USA & six \\
\hline 84 & BANG-IU & USA & six \\
\hline 85 & BRABHVTIB & USA & six \\
\hline 86 & H.VULJ.L.TRIF & USA & six \\
\hline 87 & M66.85-BI12168 & USA & six \\
\hline 88 & ORE"S"BBB-177 & USA & six \\
\hline 89 & IBNBF8-582SEL.6AP & USA & six \\
\hline 90 & IBNBF8-588SEL.1AP & USA & six \\
\hline 91 & IBNBF8-594SEL.2AP & USA & six \\
\hline 92 & FB0598 & USA & six \\
\hline 93 & FB0604 & USA & six \\
\hline 94 & FB0605 & USA & six \\
\hline 95 & FB0606 & USA & six \\
\hline 96 & FB0607 & USA & six \\
\hline 97 & FB0611 & USA & six \\
\hline 98 & FB0612 & USA & six \\
\hline 99 & FB0613 & USA & six \\
\hline 100 & FB0614 & USA & six \\
\hline 101 & FB0615 & USA & six \\
\hline 102 & FB0616 & USA & six \\
\hline 103 & FB0619 & USA & six \\
\hline 104 & JNABBB-204 & USA & six \\
\hline 105 & JNABBB-205 & USA & six \\
\hline 106 & FB0844 & USA & six \\
\hline 107 & FB0609 & USA & two \\
\hline 108 & XQ0758 & USA & $\operatorname{six}$ \\
\hline 109 & 31721 & USA & two \\
\hline 110 & GY135 & USA & six \\
\hline 111 & GY137 & USA & six \\
\hline 112 & GY138 & USA & six \\
\hline
\end{tabular}

Among the 319 SSR markers, 147 markers with clear and sharp PCR bands were selected for further analysis of genetic diversity and association mapping in this study (Table 2).

\section{Statistical Analysis}

Analysis of variance (ANOVA) for the phenotypic data was carried out using the general linear model function (GLM) of the SPSS statistical package (SPSS Inc., Chicago, IL). The genotypic variance $\mathrm{V}_{\mathrm{g}}^{2}$, genotypeenvironment interaction $\mathrm{V}^{2}$ ge, and environmental variances $\mathrm{V}_{\mathrm{e}}^{2}$ were estimated for each trait to measure their heritability $\left(h_{\mathrm{m}}^{2}\right)$ by the following formula: $h^{2} \mathrm{~m}=$ $\mathrm{V}^{2} \mathrm{~g} /\left(\mathrm{V}^{2}{ }_{\mathrm{g}}+\mathrm{V}^{2}{ }_{\mathrm{ge}} / e+\mathrm{V}^{2}{ }_{\mathrm{e}} / r e\right)$, where $e$ is number of trials and $r$ is number of replications (Long et al., 2013). 
Lai et al. / Int. J. Agric. Biol., Vol. 19, No. 5, 2017

Table 2: Number of alleles and PIC values caculated based on the panel of 112 barley genotypes

\begin{tabular}{|c|c|c|c|c|c|c|c|}
\hline$\overline{\text { Code }}$ & Locus & Chromosome & $\begin{array}{l}\text { Position }^{\mathrm{a}} \\
\end{array}$ & Annealing Temperature $\left({ }^{\circ} \mathrm{C}\right)$ & $\mathrm{Na}^{\mathrm{b}}$ & $\mathrm{Ne}^{\mathrm{c}}$ & $\mathrm{PIC}^{\mathrm{d}}$ \\
\hline 1 & EBmac0560 & $1 \mathrm{HS}$ & 58.76 & 55 & 2 & 1.02 & 0.0177 \\
\hline 2 & GBM1412 & 1HS & 59.11 & 55 & 2 & 1.03 & 0.0351 \\
\hline 3 & Bmag0350 & 1HS & 59.83 & 55 & 10 & 6.21 & 0.8388 \\
\hline 4 & Bmag0211 & $1 \mathrm{HS}$ & 60.42 & 60 & 6 & 4.41 & 0.7730 \\
\hline 5 & $G B M 1451$ & 1HS & 60.52 & 55 & 5 & 2.61 & 0.6157 \\
\hline 6 & GBM1234 & 1HS & 61.06 & 55 & 2 & 1.55 & 0.3565 \\
\hline 7 & EBmac0659 & 1HS & 61.81 & 55 & 2 & 1.02 & 0.0177 \\
\hline 8 & GBM1336 & $1 \mathrm{HL}$ & 63.01 & 55 & 2 & 1.06 & 0.0605 \\
\hline 9 & Bmac0090 & $1 \mathrm{HL}$ & 63.66 & 55 & 10 & 6.15 & 0.8374 \\
\hline 10 & EBmac0695 & $1 \mathrm{HL}$ & 65.31 & 55 & 3 & 2.13 & 0.5311 \\
\hline 11 & HVM2O & $1 \mathrm{HL}$ & 66.25 & 55 & 5 & 3.27 & 0.6942 \\
\hline 12 & $\operatorname{scssr} 10477$ & $1 \mathrm{HL}$ & 79.09 & 55 & 5 & 1.32 & 0.2430 \\
\hline 13 & GBM1092 & $1 \mathrm{HL}$ & 91.79 & 55 & 1 & 1.00 & 0.0000 \\
\hline 14 & GBM5162 & $1 \mathrm{HL}$ & 93.12 & 55 & 2 & 1.02 & 0.0177 \\
\hline 15 & Bmag0579 & $1 \mathrm{HL}$ & 132.84 & 55 & 8 & 2.63 & 0.6202 \\
\hline 16 & GBM1461 & $1 \mathrm{HL}$ & 135.94 & 55 & 6 & 4.09 & 0.7554 \\
\hline 17 & $G B M 1434$ & $1 \mathrm{HL}$ & 136.38 & 55 & 2 & 1.87 & 0.4641 \\
\hline 18 & scssr 08238 & $1 \mathrm{HL}$ & 139.81 & 55 & 5 & 1.98 & 0.4949 \\
\hline 19 & GBM1204 & $1 \mathrm{HL}$ & 139.81 & 55 & 3 & 2.67 & 0.5594 \\
\hline 20 & GBM1187 & 2HS & 19.51 & 55 & 2 & 1.72 & 0.4193 \\
\hline 21 & scssr 10226 & $2 \mathrm{HS}$ & 46.63 & 55 & 3 & 1.81 & 0.4456 \\
\hline 22 & scssr07759 & 2HS & 48.58 & 55 & 4 & 1.74 & 0.4245 \\
\hline 23 & GBM1446 & $2 \mathrm{HS}$ & 58.13 & 55 & 3 & 1.68 & 0.4033 \\
\hline 24 & $G B M 1251$ & $2 \mathrm{HS}$ & 58.8 & 55 & 5 & 2.79 & 0.6427 \\
\hline 25 & $H v X a n$ & 2HS & 62.69 & 55 & 2 & 1.29 & 0.2254 \\
\hline 26 & $\operatorname{scssr} 03381$ & $2 \mathrm{HS}$ & 63.61 & 55 & 5 & 4.51 & 0.7779 \\
\hline 27 & $G B M 1459$ & 2HS & 64.35 & 55 & 5 & 4.29 & 0.7671 \\
\hline 28 & GBM5230 & $2 \mathrm{HS}$ & 65.16 & 55 & 2 & 1.09 & 0.0853 \\
\hline 29 & GMS003 & $2 \mathrm{HS}$ & 66.05 & 60 & 7 & 1.64 & 0.3887 \\
\hline 30 & EBmac0640 & $2 \mathrm{HS}$ & 68.78 & 55 & 4 & 1.17 & 0.1429 \\
\hline 31 & GBM1203 & $2 \mathrm{HS}$ & 69.58 & 55 & 2 & 1.11 & 0.1014 \\
\hline 32 & EBmac0525 & $2 \mathrm{HS}$ & 70.98 & 55 & 1 & 1.00 & 0.0000 \\
\hline 33 & Bmag0518 & $2 \mathrm{HL}$ & 72.01 & 60 & 11 & 5.04 & 0.8015 \\
\hline 34 & Bmag0829 & $2 \mathrm{HL}$ & 74.56 & 55 & 6 & 2.65 & 0.6229 \\
\hline 35 & Bmag0711 & $2 \mathrm{HL}$ & 79.52 & 55 & 8 & 3.33 & 0.6993 \\
\hline 36 & GBM1468 & $2 \mathrm{HL}$ & 84.06 & 55 & 4 & 1.83 & 0.4538 \\
\hline 37 & GBM1408 & $2 \mathrm{HL}$ & 89.44 & 55 & 2 & 1.13 & 0.1172 \\
\hline 38 & EBmatc0039 & $2 \mathrm{HL}$ & 93.26 & 60 & 3 & 1.39 & 0.2801 \\
\hline 39 & GBM1440 & $2 \mathrm{HL}$ & 96.34 & 55 & 4 & 2.67 & 0.5594 \\
\hline 40 & GBM1208 & $2 \mathrm{HL}$ & 102.85 & 55 & 5 & 2.31 & 0.5669 \\
\hline 41 & $G B M 1149$ & $2 \mathrm{HL}$ & 107.89 & 55 & 4 & 2.61 & 0.6172 \\
\hline 42 & EBmac0415 & $2 \mathrm{HL}$ & 117.86 & 55 & 4 & 1.93 & 0.4831 \\
\hline 43 & GBM1200 & $2 \mathrm{HL}$ & 124.8 & 55 & 1 & 1.00 & 0.0000 \\
\hline 44 & $G B M 1498$ & $2 \mathrm{HL}$ & 125.71 & 55 & 3 & 2.32 & 0.5662 \\
\hline 45 & Bmag0749 & $2 \mathrm{HL}$ & 147.93 & 55 & 5 & 2.69 & 0.6284 \\
\hline 46 & GBM1475 & $2 \mathrm{HL}$ & 149.47 & 55 & 3 & 1.59 & 0.3692 \\
\hline 47 & scssr 08447 & $2 \mathrm{HL}$ & 156.49 & 55 & 5 & 2.55 & 0.6079 \\
\hline 48 & $G B M 1280$ & $3 \mathrm{HS}$ & 3.83 & 55 & 2 & 1.43 & 0.2991 \\
\hline 49 & GBM1450 & $3 \mathrm{HS}$ & 13.94 & 55 & 2 & 1.39 & 0.2817 \\
\hline 50 & GBM1382 & $3 \mathrm{HS}$ & 16.32 & 55 & 4 & 3.82 & 0.7384 \\
\hline 51 & EBmac0705 & $3 \mathrm{HS}$ & 20.49 & 55 & 5 & 3.01 & 0.6676 \\
\hline 52 & $\operatorname{scssr} 10559$ & $3 \mathrm{HS}$ & 23.26 & 55 & 6 & 1.94 & 0.4846 \\
\hline 53 & $G B M 1284$ & $3 \mathrm{HS}$ & 31.95 & 55 & 2 & 1.05 & 0.0436 \\
\hline 54 & Bmag0023 & $3 \mathrm{HS}$ & 46.28 & 55 & 1 & 1.00 & 0.0000 \\
\hline 55 & GBM1163 & $3 \mathrm{HS}$ & 60.27 & 55 & 2 & 1.09 & 0.0853 \\
\hline 56 & GBM1110 & $3 \mathrm{HS}$ & 60.27 & 55 & 3 & 2.19 & 0.5429 \\
\hline 57 & $G B M 1495$ & $3 \mathrm{HL}$ & 62.57 & 55 & 2 & 1.11 & 0.1014 \\
\hline 58 & GBM1405 & $3 \mathrm{HL}$ & 86.33 & 55 & 4 & 1.51 & 0.3367 \\
\hline 59 & $G B M 1233$ & $3 \mathrm{HL}$ & 89.66 & 55 & 3 & 1.61 & 0.3774 \\
\hline 60 & Bmag0013 & $3 \mathrm{HL}$ & 113.7 & 60 & 12 & 6.35 & 0.8426 \\
\hline 61 & $G B M 1420$ & $3 \mathrm{HL}$ & 152.53 & 55 & 5 & 3.28 & 0.6955 \\
\hline 62 & GBM1501 & 4HS & 0 & 55 & 2 & 1.52 & 0.3418 \\
\hline 63 & $H V M 40$ & $4 \mathrm{HS}$ & 22.4 & 60 & 8 & 3.22 & 0.6894 \\
\hline 64 & $G B M 1323$ & 4HS & 28.96 & 55 & 3 & 1.88 & 0.4686 \\
\hline 65 & scssr 20569 & 4HS & 44.87 & 55 & 4 & 1.84 & 0.4579 \\
\hline
\end{tabular}


Association Mapping of Grain Size in Barley / Int. J. Agric. Biol., Vol. 19, No. 5, 2017

Table 2: Continued

\begin{tabular}{|c|c|c|c|c|c|c|c|}
\hline 66 & Bmag0808 & $4 \mathrm{HL}$ & 53.04 & 55 & 7 & 3.43 & 0.7082 \\
\hline 67 & EВтас0906 & $4 \mathrm{HL}$ & 54.98 & 55 & 5 & 1.87 & 0.4650 \\
\hline 68 & Втас0181 & $4 \mathrm{HL}$ & 58.51 & 55 & 5 & 3.45 & 0.7098 \\
\hline 69 & Bmac0030 & $4 \mathrm{HL}$ & 58.6 & 55 & 9 & 3.21 & 0.6876 \\
\hline 70 & Bmag0490 & $4 \mathrm{HL}$ & 62.19 & 55 & 11 & 3.13 & 0.6809 \\
\hline 71 & GBM1299 & $4 \mathrm{HL}$ & 72.04 & 60 & 3 & 1.37 & 0.2686 \\
\hline 72 & EBmac0658 & $4 \mathrm{HL}$ & 75.59 & 55 & 4 & 1.81 & 0.4466 \\
\hline 73 & EВmac0635 & $4 \mathrm{HL}$ & 93.06 & 55 & 11 & 2.26 & 0.5582 \\
\hline 74 & EВтас0679 & $4 \mathrm{HL}$ & 94.5 & 55 & 10 & 2.48 & 0.5974 \\
\hline 75 & EBmac0701 & $4 \mathrm{HL}$ & 96.15 & 55 & 15 & 2.61 & 0.6159 \\
\hline 76 & GBM1220 & $4 \mathrm{HL}$ & 99.86 & 55 & 4 & 1.33 & 0.2496 \\
\hline 77 & HVMLOHIA & $4 \mathrm{HL}$ & 102.27 & 55 & 4 & 2.06 & 0.5148 \\
\hline 78 & HVM67 & $4 \mathrm{HL}$ & 120.5 & 60 & 4 & 1.98 & 0.4950 \\
\hline 79 & $G B M 1388$ & $4 \mathrm{HL}$ & 122.35 & 55 & 2 & 1.93 & 0.4807 \\
\hline 80 & Bmag0138 & $4 \mathrm{HL}$ & 124 & 55 & 6 & 2.69 & 0.6276 \\
\hline 81 & GBM1453 & $4 \mathrm{HL}$ & 132.27 & 55 & 3 & 1.88 & 0.4668 \\
\hline 82 & scssr02306 & $5 \mathrm{HS}$ & 6.13 & 55 & 3 & 2.51 & 0.6022 \\
\hline 83 & GBM1176 & $5 \mathrm{HS}$ & 18.59 & 55 & 2 & 1.44 & 0.3047 \\
\hline 84 & scssr07106 & $5 \mathrm{HS}$ & 20.44 & 55 & 4 & 1.86 & 0.4635 \\
\hline 85 & GBM5028 & $5 \mathrm{HS}$ & 27.41 & 55 & 3 & 1.61 & 0.3793 \\
\hline 86 & EBmac0970 & $5 \mathrm{HS}$ & 40.77 & 55 & 2 & 1.96 & 0.4885 \\
\hline 87 & Hvm30 & $5 \mathrm{HS}$ & 41.93 & 55 & 4 & 1.49 & 0.3327 \\
\hline 88 & Bmag0751 & $5 \mathrm{HS}$ & 42.87 & 55 & 10 & 3.77 & 0.7346 \\
\hline 89 & Bmag0337 & $5 \mathrm{HS}$ & 44.99 & 55 & 8 & 3.68 & 0.7285 \\
\hline 90 & Bmag0323 & $5 \mathrm{HS}$ & 50.85 & 55 & 14 & 11.06 & 0.9096 \\
\hline 91 & Bmac0096 & $5 \mathrm{HS}$ & 53.12 & 60 & 6 & 3.64 & 0.7251 \\
\hline 92 & GBM1399 & $5 \mathrm{HL}$ & 69.86 & 55 & 3 & 1.77 & 0.4342 \\
\hline 93 & EBmac0684 & $5 \mathrm{HL}$ & 73.79 & 55 & 6 & 2.27 & 0.5585 \\
\hline 94 & GBM1506 & $5 \mathrm{HL}$ & 75.45 & 55 & 6 & 2.13 & 0.5312 \\
\hline 95 & scssr 15334 & $5 \mathrm{HL}$ & 77.79 & 55 & 6 & 2.19 & 0.5444 \\
\hline 96 & GBM1483 & $5 \mathrm{HL}$ & 80.64 & 55 & 2 & 1.11 & 0.1014 \\
\hline 97 & Bmag0812 & $5 \mathrm{HL}$ & 90.32 & 55 & 10 & 4.62 & 0.7835 \\
\hline 98 & scssr05939 & $5 \mathrm{HL}$ & 90.64 & 55 & 3 & 1.67 & 0.4022 \\
\hline 99 & GBM1227 & $5 \mathrm{HL}$ & 91.82 & 55 & 2 & 1.19 & 0.1626 \\
\hline 100 & $G B M 1231$ & $5 \mathrm{HL}$ & 102.27 & 55 & 3 & 1.15 & 0.1270 \\
\hline 101 & GBM1438 & $5 \mathrm{HL}$ & 104.74 & 55 & 3 & 1.35 & 0.2616 \\
\hline 102 & GBM1295 & $5 \mathrm{HL}$ & 107.11 & 55 & 3 & 2.47 & 0.5945 \\
\hline 103 & GBM5008 & $5 \mathrm{HL}$ & 118.98 & 55 & 4 & 1.53 & 0.3479 \\
\hline 104 & GBM1363 & $5 \mathrm{HL}$ & 120.68 & 55 & 2 & 1.36 & 0.2637 \\
\hline 105 & GMS061 & $5 \mathrm{HL}$ & 122.09 & 60 & 8 & 3.76 & 0.7339 \\
\hline 106 & EBmatc 0003 & $5 \mathrm{HL}$ & 127.69 & 60 & 4 & 2.19 & 0.5452 \\
\hline 107 & GBM1470 & $5 \mathrm{HL}$ & 130.55 & 55 & 1 & 1.00 & 0.0000 \\
\hline 108 & GBM1166 & $5 \mathrm{HL}$ & 133.5 & 55 & 2 & 1.32 & 0.2449 \\
\hline 109 & GBM1490 & $5 \mathrm{HL}$ & 144.14 & 55 & 3 & 1.97 & 0.493 \\
\hline 110 & GBM1164 & $5 \mathrm{HL}$ & 156.88 & 55 & 2 & 1.09 & 0.0853 \\
\hline 111 & $84 c 21 j 33$ & $6 \mathrm{HS}$ & 0 & 55 & 4 & 2.93 & 0.6585 \\
\hline 112 & Bmac0316 & $6 \mathrm{HS}$ & 7.16 & 55 & 6 & 1.42 & 0.2970 \\
\hline 113 & GBM1270 & $6 \mathrm{HS}$ & 36.52 & 55 & 2 & 1.23 & 0.2052 \\
\hline 114 & GBM1215 & $6 \mathrm{HS}$ & 39.54 & 55 & 6 & 2.69 & 0.6283 \\
\hline 115 & $G B M 1212$ & $6 \mathrm{HS}$ & 55.1 & 55 & 3 & 1.41 & 0.2929 \\
\hline 116 & Bmac0040 & $6 \mathrm{HS}$ & 61.07 & 55 & 7 & 2.14 & 0.5330 \\
\hline 117 & Bmag0344 & $6 \mathrm{HS}$ & 67.8 & 55 & 1 & 1.00 & 0.0000 \\
\hline 118 & scssr05599 & $6 \mathrm{HL}$ & 96.34 & 55 & 4 & 3.12 & 0.6797 \\
\hline 119 & GBM1140 & $6 \mathrm{HL}$ & 97.31 & 55 & 2 & 1.99 & 0.4998 \\
\hline 120 & $G B M 1274$ & $6 \mathrm{HL}$ & 123.45 & 55 & 1 & 1.00 & 0.0000 \\
\hline 121 & GBM1276 & $6 \mathrm{HL}$ & 124.29 & 55 & 2 & 1.93 & 0.4824 \\
\hline 122 & GBM1275 & $6 \mathrm{HL}$ & 124.29 & 55 & 3 & 1.98 & 0.4959 \\
\hline 123 & GBM1087 & $6 \mathrm{HL}$ & 127.7 & 55 & 3 & 2.97 & 0.6632 \\
\hline 124 & GBM1404 & $6 \mathrm{HL}$ & 129.76 & 55 & 1 & 1.00 & 0.0000 \\
\hline 125 & GBM1126 & $7 \mathrm{HS}$ & 8.8 & 55 & 4 & 2.67 & 0.6258 \\
\hline 126 & Bmag0206 & $7 \mathrm{HS}$ & 15.25 & 55 & 12 & 5.61 & 0.8216 \\
\hline 127 & GBM5060a & $7 \mathrm{HS}$ & unknown & 55 & 1 & 1.00 & 0.0000 \\
\hline 128 & GBM1326a & $7 \mathrm{HS}$ & unknown & 55 & 1 & 1.00 & 0.0000 \\
\hline 129 & GBM5060b & $7 \mathrm{HS}$ & 31.24 & 55 & 7 & 3.42 & 0.7079 \\
\hline 130 & $G B M 1326 b$ & $7 \mathrm{HS}$ & 31.24 & 55 & 7 & 3.58 & 0.7208 \\
\hline 131 & EВтас0603 & $7 \mathrm{HS}$ & 35.39 & 60 & 5 & 1.74 & 0.4267 \\
\hline
\end{tabular}


Table 2: Continued

\begin{tabular}{|c|c|c|c|c|c|c|c|}
\hline 132 & Bmag0914 & 7HS & 46.85 & 55 & 7 & 2.32 & 0.5686 \\
\hline 133 & GBM1464 & 7HS & 53.43 & 60 & 6 & 1.93 & 0.4824 \\
\hline 134 & Bmac0282 & 7HS & 59.31 & 55 & 1 & 1.00 & 0.0000 \\
\hline 135 & Bmac0187 & 7HS & 72.57 & 60 & 8 & 3.43 & 0.7080 \\
\hline 136 & GBM1432 & 7HS & 72.81 & 55 & 1 & 1.00 & 0.0000 \\
\hline 137 & Bmag0321 & $7 \mathrm{HL}$ & 79.24 & 60 & 9 & 4.09 & 0.7559 \\
\hline 138 & EBmac0827 & $7 \mathrm{HL}$ & 80.57 & 55 & 8 & 2.93 & 0.6584 \\
\hline 139 & $G B M 1115 a$ & $7 \mathrm{HL}$ & unknown & 55 & 1 & 1.00 & 0.0000 \\
\hline 140 & $G B M 1115 b$ & $7 \mathrm{HL}$ & 81.85 & 55 & 5 & 4.48 & 0.7767 \\
\hline 141 & Bmag0369 & $7 \mathrm{HL}$ & 83.31 & 60 & 5 & 1.91 & 0.4749 \\
\hline 142 & GBM1303 & $7 \mathrm{HL}$ & 86.43 & 55 & 9 & 3.78 & 0.7358 \\
\hline 143 & GBM1297 & $7 \mathrm{HL}$ & 88.56 & 55 & 2 & 1.46 & 0.3157 \\
\hline 144 & GBM1174 & $7 \mathrm{HL}$ & 93.86 & 55 & 2 & 1.25 & 0.1983 \\
\hline 145 & GBM5225 & $7 \mathrm{HL}$ & 101.73 & 55 & 3 & 2.61 & 0.6171 \\
\hline 146 & GBM1456 & $7 \mathrm{HL}$ & 136.79 & 55 & 2 & 1.65 & 0.3922 \\
\hline 147 & $\operatorname{scssr} 04056$ & 7HL & 148.22 & 55 & 11 & 4.18 & 0.7608 \\
\hline
\end{tabular}

Pearson correlation coefficients between grain weight, grain length and grain width in different field trials were calculated using SPSS.

For each marker, its genetic diversity parameters, observed number of alleles and effective number of alleles per locus were calculated using POPGENE software version 1.32 (Yeh and Boyle, 1999). Additionally, Polymorphism information content (PIC) values were computed by the formula: PIC $=1-\sum P_{i}^{2}$, where $P_{i}$ is the frequency of the $i^{\text {th }}$ SSR allele, with the software PowerMarker version 3.25 (Liu and Muse, 2005).

\section{Population Structure Estimate}

The population structure of the diversity panel was investigated based on a set of 28 unlinked SSR markers, which were located on different chromosome arms. The genetic distance between two chosen markers on the same chromosome arm was more than $20 \mathrm{cM}$ to avoid genetic linkage (Kulwal et al., 2012). Population structure was estimated using principal component analysis (PCA) based on simple matching of alleles performed by software TASSEL 2.0 (Yu et al., 2006) and by the Bayesian clustering analysis with STRUCTURE software version 2.3.3 (Pritchard et al., 2000) running 10 times independently, with $K$ ranging from 1 to 11 in each run using default setting of admixture model for the ancestry of individual and correlated allele frequencies. To confirm the true number of subgroups $(K), \Delta K$ calculated as described by Evanno et al. (2005) was plotted against $K$ for the second approach. The best number of population subgroup was determined by $\Delta K$ with their peaking value (Evanno et al., 2005).

\section{Association Analysis}

Association analysis was carried out using mixed linear model (MLM) approach as described in the software package TASSEL 2.0 (Bradbury et al., 2007). SSR markers with minor allele frequency less than 5\% were removed from the data set to reduce false associations. Furthermore, to control both Type I and Type II errors, $Q$ values obtained by the software STRUCTURE and kinship matrix generated using the program TASSEL 2.0 were implemented as covariates in MLM analysis (Bradbury et al., 2007).

A threshold of $\mathrm{P}=0.01\left[-\log _{10}(\mathrm{P})=2\right]$ was set for declaring the significant marker-trait associations. Significant markers representing the same QTL were defined as having a linkage distance less than $12 \mathrm{cM}$ and $\mathrm{LD}$ (measured as $r^{2}$ from pair-wise analysis) greater than 0.2 (Locatelli et al., 2013).

\section{Allele Effects Evaluate}

Phenotypic effects of major alleles were evaluated in comparison to the 'null allele' (plus missing and rare alleles) for each locus (Breseghello and Sorrells, 2006b), and T-test between major allele and 'null allele' was conducted using the SPSS statistical package (SPSS Inc., Chicago, IL).

\section{Results}

\section{Phenotypic Analysis}

Large phenotypic variations were observed for grain weight, grain length and grain width in different trials, and the phenotype values of each trait followed normal distribution in the population (Table 3), indicating a multigene genetic programming model. The heritability $\left(h_{\mathrm{m}}^{2}\right)$ for grain weight, grain lenth and grain width across the three environments were $83.6 \%, 70.0 \%$ and $69.9 \%$, respectively (Table 3), suggesting the grain length and grain width were more sensitive to environment than grain weight. 
ANOVA also showed that the mean square (MS) values of environment $(\mathrm{E})$ for grain length and grain width were much greater than their MSs of genotype $(\mathrm{G})$ (Table 4). Moreover, $\mathrm{G} \times \mathrm{E}$ interaction (GEI) variances were significant for grain weight, length and width $(\mathrm{P}<0.01)$ reflecting that different genotypes had different sensitivity to environmental alternatives. However, their values of MS were often less than $G$ and E (Table 4). Positive correlations were detected among these traits (Table 5). The average of correlation coefficient between grain weight and grain width was greater than that between grain weight and grain length across multienvironments (Table 4). It seemed that grain width had stronger influence on grain weight.

\section{Genetic Diversity}

In this study a total of 147 SSR markers distributed on all seven chromosomes were screened to evaluate the panel diversity, with 19 markers on the chromosome $1 \mathrm{H}, 28$ on $2 \mathrm{H}, 14$ on $3 \mathrm{H}, 20$ on $4 \mathrm{H}, 29$ on $5 \mathrm{H}, 14$ on $6 \mathrm{H}$ and 23 on $7 \mathrm{H}$. Most of the loci detected for the SSR markers were distributed on short and long chromosome arm uniformly, except that 16 out of 20 loci were clustered on the long arm of chromosome 4H. Diversity statistics calculated for polymorphic locus were summarized in Supplementary Table 2. The total number of alleles ranged from 1 to 15 , with an average of 4.5 alleles per locus. The effective number of alleles varied from 1.0 to 11.1 , with an average of 2.3. The monomorphic loci, such as GBM1092, GBM1200, EBmac0525, Bmag0023, GBM1470, GBM1274, GBM1404, Bmag0344, GBM1115a, GBM1432, GBM5060a, GBM1326a and Bmac0282, were excluded and the rest ones were selected for further AM analysis. For the left 134 loci, PIC value was estimated from 0.0177 to 0.9086 (Bmag0323). The average PIC values of the seven chromosomes ranged from $0.3883(6 \mathrm{H})$ to $0.5265(4 \mathrm{H})$ (Fig. 5).

\section{Population Structure}

The model-based Bayesian clustering showed that the average $\operatorname{Ln}[\mathrm{P}(\mathrm{D})]$ (log-probability of data) value increased continuously with $K$ increasing from 1 to 11 , and the most apparent inflection was obtained when $K$ was 2 (Fig. 6). The result of subgroup numbers $(K)$ was further inferred using $\Delta K$ estimation, and the maximum peak value of $\Delta K$ was also obtained at $K=2$ (Fig. 1). Thus, the panel of 112 barley accessions was divided into two distinct subgroups using model-based Bayesian clustering. Interestingly, principal component analysis strong supported the similar result that the first subgroup accessions mostly originated from East Asia (Fig. 2), in which all of them are hull-less barley, while the second subgroup accessions were mostly from North America (Fig. 2), in which most of them are hulled barley except for the accessions WAS3, LDLQK, MZQK, B3034
Table 3: Phenotypic distribution and heritability of grain weight, grain length and grain width in the AM panel

\begin{tabular}{llllllc}
\hline Trait & Trial & Mean \pm S.E. & \multicolumn{2}{c}{ Range } & Asymp. Sig. ${ }^{\text {a }} h_{\mathrm{m}}{ }^{2}(\%)$ \\
\cline { 4 - 6 } & & & Max & Min & & \\
\hline TGW (g) & CD2014 & $27.9 \pm 0.5$ & 41.6 & 13.5 & 0.887 & 83.6 \\
& CD2015 & $29.1 \pm 0.5$ & 45.4 & 17.9 & 0.949 & \\
& NC2015 & $28.7 \pm 0.6$ & 43.6 & 14.5 & 0.593 & \\
GL (mm) & CD2014 & $6.42 \pm 0.07$ & 8.03 & 4.82 & 0.998 & 70.7 \\
& CD2015 & $6.45 \pm 0.07$ & 8.06 & 4.56 & 0.852 & \\
NC2015 & $6.88 \pm 0.07$ & 8.76 & 4.99 & 0.817 & \\
GW (mm) & CD2014 & $2.59 \pm 0.03$ & 3.21 & 1.57 & 0.636 & 69.9 \\
& CD2015 & $2.84 \pm 0.03$ & 3.44 & 2.02 & 0.983 & \\
& NC2015 & $2.82 \pm 0.03$ & 3.51 & 1.94 & 0.933 & \\
\hline
\end{tabular}

TGW, grain weight; GL grain length; GW, grain width

${ }^{a}$ Two-tail and Kolmogorov-Smirnov test for normal distribution

Table 4: ANOVA analyses of grain weight, grain length and grain width in multi-environment trials

\begin{tabular}{|c|c|c|c|c|}
\hline Trait & Source of variation & DF & MS & $\mathrm{MS} \%^{\mathrm{a}}$ \\
\hline \multirow[t]{3}{*}{ TGW } & Genotype (G) & 111 & $146.44 * *$ & 53.92 \\
\hline & Environment (E) & 2 & $87.22^{* * *}$ & 32.12 \\
\hline & GEI & 222 & $37.90 * *$ & 13.96 \\
\hline \multirow[t]{3}{*}{ GL } & Genotype (G) & 111 & $1.55 * *$ & 8.89 \\
\hline & Environment (E) & 2 & $15.00 * *$ & 86.53 \\
\hline & GEI & 222 & $0.85 * *$ & 4.86 \\
\hline \multirow[t]{3}{*}{ GW } & Genotype (G) & 111 & $0.29 * *$ & 5.94 \\
\hline & Environment (E) & 2 & $4.36 * *$ & 90.92 \\
\hline & GEI & 222 & $0.15 * *$ & 3.15 \\
\hline
\end{tabular}

Table 5: Correlation coefficients among grain weight, grain length and grain width in different trials

\begin{tabular}{lll}
\hline Traits & Grain length & Grain width \\
\hline Grain weight & $0.599 * *$ & $0.588^{* *}$ \\
& $0.378 * *$ & $0.523 * *$ \\
& $0.264 * *$ & $0.687 * *$ \\
Grain lenth & $0.673 * *$ \\
& $0.579 * *$ \\
& $0.581 * *$ \\
\hline TFor each trait, the correlation coefficients from CD2014, CD2015 \\
and NC2015 are represented in the first, second and third rows, \\
respectively \\
$* *$ indicate significance at $\mathrm{P}<0.01$
\end{tabular}

and Tibetannia. All of genotypes in the first subgroup are six-row barley except accession "K5", while most of two-row barleys are clustered into the second subgroup of North America.

\section{Association Mapping}

Marker-trait associations were identified using the $\mathrm{Q}+\mathrm{K}$ model. A significant QTL was declared when the P value for marker-trait association was less than 0.01 [$\left.\log _{10}(\mathrm{P})=2\right]$ in the QTL regions. A total of 11 QTLs were detected for three traits investigated in this study across three environments (Table 6). 
Table 6: Markers showing significant association with grain weight, grain length and grain width in mixed linear model $(\mathrm{Q}+\mathrm{K})$

\begin{tabular}{|c|c|c|c|c|c|c|c|c|c|}
\hline \multirow[t]{2}{*}{ Trait } & \multirow[t]{2}{*}{ Locus } & \multirow[t]{2}{*}{ Chr. } & \multirow[t]{2}{*}{ Pos. (cM) } & \multicolumn{2}{|c|}{ CD2014 } & \multicolumn{2}{|c|}{ CD2015 } & \multicolumn{2}{|c|}{ NC2015 } \\
\hline & & & & $-\log _{10}(\mathrm{P})$ & $R^{2}(\%)$ & $-\log _{10}(\mathrm{P})$ & $R^{2}(\%)$ & $-\log _{10}(\mathrm{P})$ & $R^{2}(\%)$ \\
\hline \multirow[t]{6}{*}{ Grain weight } & Bmag0579 & $1 \mathrm{HL}$ & 132.84 & 2.1 & 2.71 & - & - & - & - \\
\hline & scssr08238 & $1 \mathrm{HL}$ & 139.81 & 2.2 & 2.75 & - & - & - & - \\
\hline & scssr 10226 & $2 \mathrm{HS}$ & 46.63 & - & - & - & - & 2.0 & 2.73 \\
\hline & $G B M 1468$ & $2 \mathrm{HL}$ & 84.06 & - & - & - & - & 2.5 & 4.16 \\
\hline & GBM1408 & $2 \mathrm{HL}$ & 89.44 & - & - & - & - & 2.1 & 2.76 \\
\hline & GBM5028 & $5 \mathrm{HS}$ & 27.41 & 2.4 & 2.60 & 2.8 & 2.08 & - & - \\
\hline \multirow[t]{4}{*}{ Grain length } & HVMLOHIA & $4 \mathrm{HL}$ & 102.27 & - & - & 2.2 & 4.17 & - & - \\
\hline & GBM5028 & $5 \mathrm{HS}$ & 27.41 & - & - & - & - & 2.1 & 2.25 \\
\hline & GBM1215 & $6 \mathrm{HS}$ & 39.54 & 4.9 & 4.49 & - & - & - & - \\
\hline & GBM1456 & $7 \mathrm{HL}$ & 136.79 & - & - & - & - & 2.0 & 2.05 \\
\hline \multirow[t]{2}{*}{ Grain width } & scssr 10226 & $2 \mathrm{HS}$ & 46.63 & - & - & 2.3 & 5.82 & 2.1 & 3.26 \\
\hline & $G B M 1468$ & $2 \mathrm{HL}$ & 84.06 & - & - & 2.0 & 5.67 & - & - \\
\hline
\end{tabular}

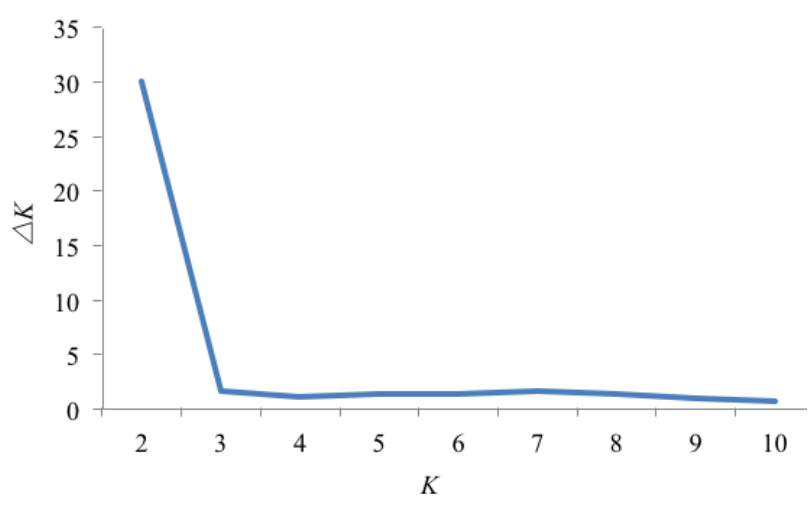

Fig. 1: The best possible subgroup number for 112 spring barley accessions using $\Delta K$ approaches

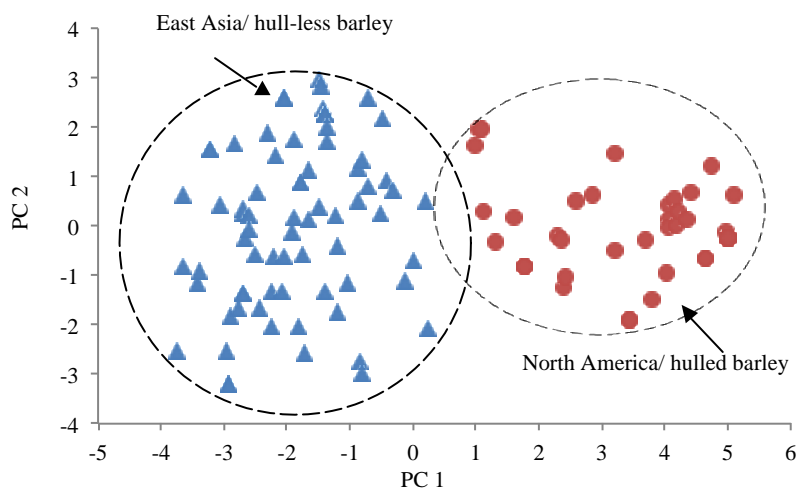

Fig. 2: Scatter plot of principal component 1 (PC1) drawn against principal component 2 (PC2). Each triangle or dot sign indicates an individual genotype in different subgroups of the AM panel. East Asia and North America accessions are represented by triangles and dots, respectively

\section{Grain Weight}

A total of five QTLs associated with seven markers were detected on chromosome arms 1HL, 2HS, 2HL, 5HS and 6HS in this study (Table 6; Fig. 3a). On 1HL, two SSR loci
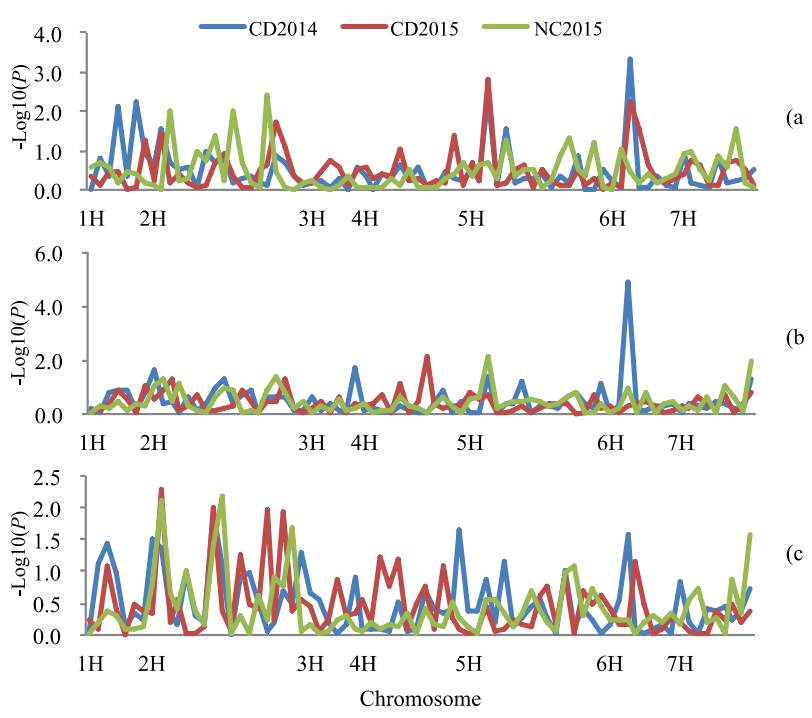

Fig. 3: Association mapping of 112 barley genotypes using the MLM for (a) grain weight, (b) grain length and (c) grain width. The $\mathrm{P}$ values were converted into $\left[-\log _{10}(\mathrm{P})\right]$

Bmag0579 and scssr08238 were significantly associated with grain weight in the CD2014 environment, and the genetic distance between these two loci was $6.97 \mathrm{cM}$ with the value of the linkage disequilibrium $\left(\mathrm{D}^{\prime}\right)$ between them was 0.85 , indicating that they were located on the same QTL region with the most significant locus scssr08238 (Table 6; Fig. 3a). On 2HL, two loci were detected to be significantly associated with grain weight in the trail of NC2015 within $5.38 \mathrm{cM}$ region (Table 6). The most significant locus was GBM1468, and thus the QTL was designated as $Q T g w$ GBM1468. The QTL on chromosome 2HS was prominent in NC2015 environment, whereas QTLs on chromosomes 5HS linked with locus GBM5028 and 6HS linked with locus GBM1215 were stable detected in both CD2014 and CD2015 environments. None of QTLs was significant in all three trials and all associated markers 
explained just a small portion of the phenotypic variation, ranging from $1.86 \%$ to $5.82 \%$, as expected for complex polygenic trait.

\section{Grain Length}

Four QTLs were found to be significantly associated with grain length (Table 6; Fig. 3b). The QTL linked with GBM1215 locus located on chromosome 6HS had the strongest associations and explained $4.49 \%$ of the phenotypic variation. Moreover, this QTL had the highest [$\left.\log _{10}(\mathrm{P})\right]$ in our study (Table 6; Fig. 3b). Four QTLs related to grain length were detected only in a single environment and also explained just a small portion of the phenotypic variation (Table 6; Fig. 3b).

\section{Grain Width}

Two QTLs on chromosome $2 \mathrm{H}$ were identified for grain width (Table 6; Fig. 3c). One QTL associated with locus scssr 10226 explained $3.26 \%$ and $5.82 \%$ of the phenotypic variation in NC2015 and CD2015 environment, respectively. Another one linked with locus GBM1468 explained $5.67 \%$ of the phenotypic variation and was detected only in CD2015 environment (Table 6; Fig. 3c).

\section{Phenotypic Effects of Major Alleles}

Among these QTLs for investigated traits, three QTLs for grain weight and width stably expressed in two environments and one QTL for grain length with the highest $\left[-\log _{10}(\mathrm{P})\right]($ Table 6$)$ were selected to evaluate phenotypic effects of their major alleles. At the locus of GBM5028 associated with grain weight, a total of two major alleles $A I$ and $A 2$ were observed and the representative accessions for them were DLH (accession code: 5) and 9516 (accession code: 4) (Table 1), respectively. Accessions carrying A2 allele produced significantly lower grain weight in CD2014 environment, which decreased about $2.5 \mathrm{~g}$ of TGW (Fig. 4a). Among the three major alleles at the locus of GBM1215 associated with grain weight, the allele of $A 4$ increased the average of $3.5 \mathrm{~g}$ of TGW significantly, while $A 3$ decreased the TGW significantly (Fig. 4a). GBM1215 was associated with grain length significantly. Among the three detected alleles, A3 decreased grain length significantly in both the trials of CD2014 and NC2015, A4 increased grain length significantly in the trials of CD2014, while the allele $A 5$ was associated with opposite effects in CD2014 and NC2015 (Fig. 4b). At the locus of scssr10226 associated with grain width, the allele $A 3$ produced significantly narrower grains, while $A l$ was significantly associated with increased grain width (Fig. 4c).

\section{Discussion}

In our study, a total of five QTLs were significantly associated with grain weight. The QTL linked to scssr 10226

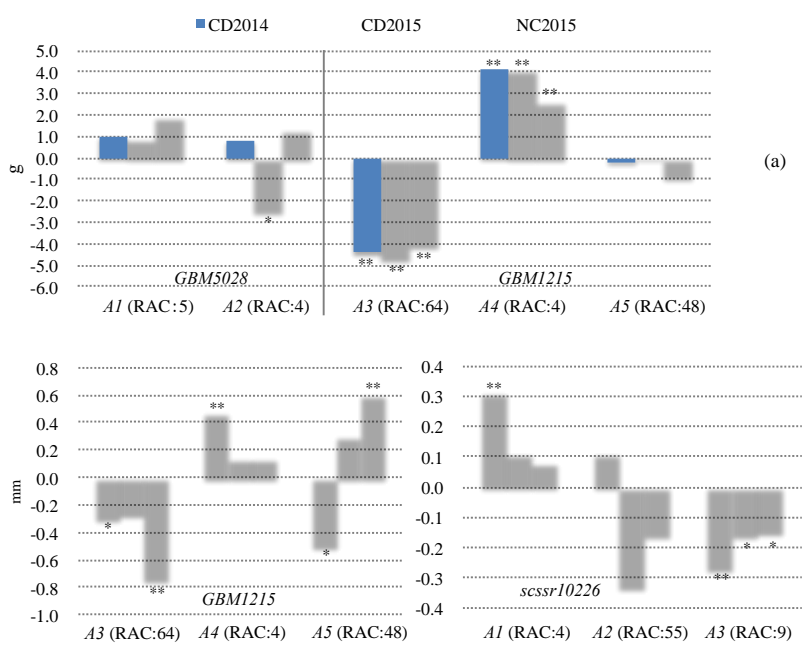

(b)

(c)

Fig. 4: Phenotypic effect of the major alleles at SSR loci associated with (a) grain weight, (b) grain length and (c) grain width in the trials of CD2014, CD2015 and NC2015. Representative accession code (RAC). *, ** mean significance of phenotypic effect of the major allele at the 0.05 and 0.01 level when comparing with 'null alleles', respectively

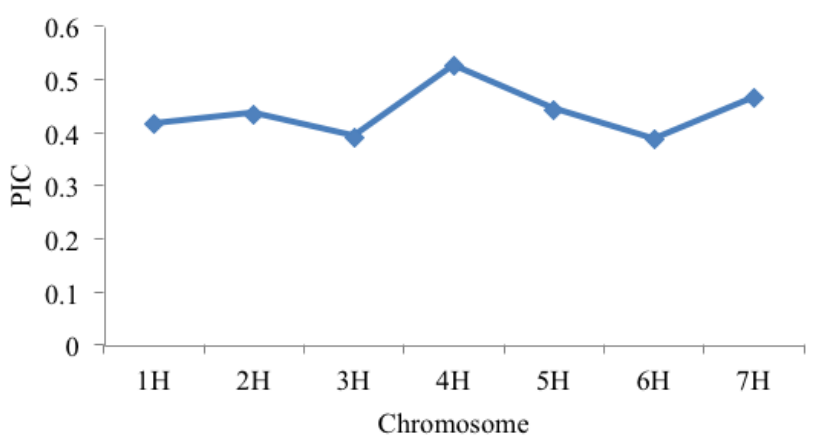

Fig. 5: Average PIC value of each chromosome in the panel of 112 barley genotypes

on the chromosome $2 \mathrm{HS}$ in this study is consistent with several reported QTLs, such as QTLs in the interval of HVM36-GMS3 (Pillen et al., 2003; Korff et al., 2006) and GBM1214 related to grain weight with $\left[-\log _{10}(\mathrm{P})\right]$ score more than 10 (Mikolajczak et al., 2016), while scssr10226 is tightly linked to GBM1214 and GMS3 based on the comparisons of genetic maps reported by Korff et al. (2004) and Varshney et al. (2007). The significant QTL QTgw-GBM1468 identified in this study on 2HL corresponded to the QTL identified by Li et al. (2005) and Bauer et al. (2009), and the QTL on chromosome 5HS in our study is in the similar genetic position to that reported by Saal et al. (2011). In addition, few QTLs are reported around the interval of QTgw-scssr08238 on chromosome $1 \mathrm{H}$. 


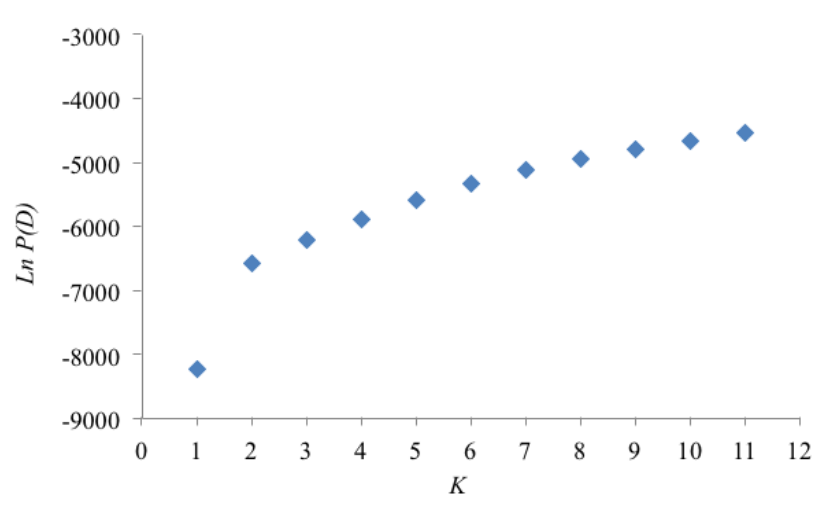

Fig. 6: Scatter plot of LnP(D) (Log probability of data), averaged over ten replications drawn against $K$. apparent inflection found at $K=2$

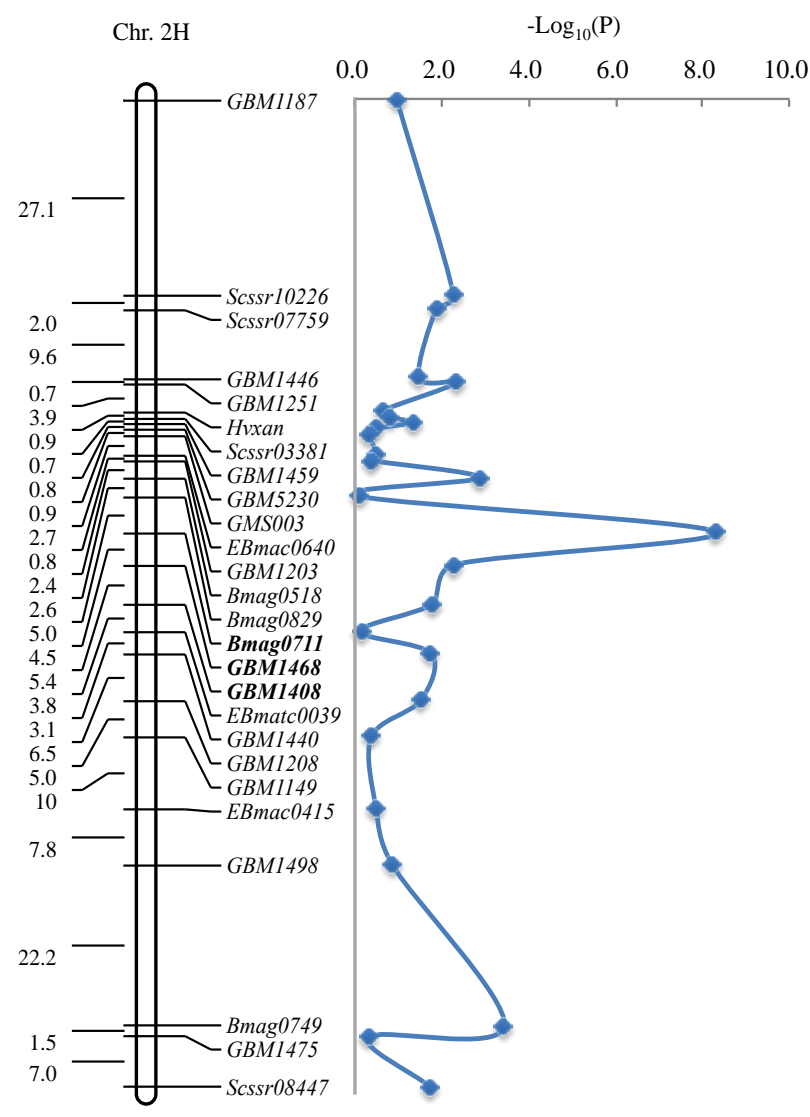

Fig. 7: Association mapping for row type on chromosome $2 \mathrm{H}$ of barley. Markers in bold indicates the interval most significantly related to row type

Among the four QTLs for grain length, the QTL on the long arm of chromosome $7 \mathrm{H}$ is similar in position to that report by Walker et al. (2013). Nevertheless, the others detected in this study seem to be different from previous reports (Backes et al., 1995; Ayoub et al., 2002; Walker et al., 2013; Zhou et al., 2016). For the two QTLs related to grain width on chromosome $2 \mathrm{H}$, one linked to the locus GBM1468 that is also significantly associated with row type (Fig. 7) is nearby locus Vrsl (Varshney et al., 2007; Huang and $\mathrm{Wu}, 2011$ ), which determines barley row type that is associated with kernel size, as QTLs associated with grain shape and weight have been detected in the interval of HVBKASI-Vrs1 (Marquezcedillo et al., 2001; Ayoub et al., 2002).

In our study, grain weight was highly positively correlated with grain length and grain width. Correlations between them partially explained the phenomenon of QTL clustering in some chromosome regions, as half of QTLs for gain length and width were co-localized with QTLs for grain weight (Table 6; Fig. 3), suggesting that grain weight was jointly influenced by both grain length and width. Walker et al. (2013) also reported that grain weight was often positively related to grain width and QTL cluster was found for both of them. However, for the grain length, grain weight was not always highly or positively correlated with it (Backes et al., 1995; Schnaithmann and Pillen, 2013). In this study, grain width was significantly positively correlated with grain length, but no QTL cluster was detected for them, indicating that grain width and grain length may be independent traits in genetic basis and the correlation between them is mostly caused by artificial selection, and this also happened in barley reported by Schnaithmann and Pillen (2013) and in wheat reported by Gegas et al. (2010).

For the SSR locus GBMS5028 associated with grain weight and grain length, $Y A B B Y$ gene family containing FAS gene (Cong et al., 2008) and YAB1, CRC,DL and INO genes (Han et al., 2015) which played important roles in the development of leaf, flower, and fruit in tomato was found to be tightly linked to it (http://webblast.ipkgatersleben.de/barley/; http://www.ncib.nlm.nih.gov/). GBM1408 associated with grain weight was linked to gene that was homologous to 1-aminocyclopropane-1carboxylate $(A C C)$ synthase gene, which regulates a number of plant processes, ranging from seed germination to organ senescence (Bleecker and Kende, 2000). For GBM1456 locus related to grain width, its linked gene $G D S L$ esterase/lipase belonged to the $\alpha / \beta$ hydrolase fold superfamily of proteins, regulating coleoptile elongation in rice as GDSL-containing enzyme rice 1 gene (GERl) (Riemann et al., 2007). Actually, the SSR primers for GBM1456 were developed from the sequence of $G D S L$ esterases/lipases gene (http://wheat.pw.usda.gov/).

For complex quantitative traits, QTLs identified by AM often explains smaller percentage of the phenotypic variation than using bi-parental mapping (Massman et al., 2011; Zhou and Steffenson, 2013; Zhou et al., 2014). In this study, the detected QTLs for grain size related traits explained no more than $6 \%$ of the phenotypic variation, which are probably caused by (1) artificial selection in cultivated germplasm pool used in our study which fixing that larger-effect QTL/gene more rapidly under the process 
of domestication or breeding, and (2) insufficient marker density in this study (Massman et al., 2011; Zhou and Steffenson, 2013).

Comparison of phenotypic effects between different QTL alleles from different germplasms is useful for breeders to choose suitable parental lines. For instance, at the site of GBM1215 associated with grain weight, if using parental lines only containing $A 3$ and $A 5$ alleles to construct bi-parental QTL mapping population, QTL allele of $A 5$ would be favor QTL allele increasing grain weight. Actually, A4 was the most interesting QTL allele for enhancing grain weight.

In conclusion, we identified eleven QTLs including five for grain weight, four for grain length and two for grain width and elite alleles improving these traits at these QTLs loci. Several QTLs identified had similar genetic regions to previous reported indicating that these QTLs might be useful for enhancing grain weight, length and width in barley breeding program.

\section{Acknowledgments}

This work was supported by Agriculture Research System in China (Grant No.: CRAS-05). We are very grateful to J. Yuan, Y. Dong and Y. Huang for conducting the field experiments.

\section{References}

Ayoub, M., S.J. Symons, M.J. Edney and D.E. Mather, 2002. QTLs affecting kernel size and shape in a two-rowed by six-rowed barley cross. Theor. Appl. Genet. 105: 237-247

Backes, G., A. Graner, B. Foroughi-Wehr, G. Fischbeck, G. Wenzel and A. Jahoor, 1995. Localization of quantitative trait loci (QTL) for agronomic important characters by the use of a RFLP map in barley (Hordeum vulgare L.). Theor. Appl. Genet., 90: 294-302

Bauer, A.M., F. Hoti, K.M. Von, K. Pillen, J. Léon and M.J. Sillanpää, 2009. Advanced backcross-QTL analysis in spring barley (H.vulgare ssp. spontaneum) comparing a REML versus a Bayesian model in muti-environmental field trials. Theor. Appl. Genet., 119: 105-123

Bezant, J., D. Laurie, N. Pratchett, J. Chojecki and M. Kearsey, 1997. Mapping QTL controlling yield and yield components in a spring barley (Hordeum vulgare L.) cross using marker regression. Mol. Breed., 3: 29-38

Bleecker, A.B. and H. Kende, 2000. Ethylene: a gaseous signal molecule in plants. Annu. Rev. Cell Dev. Biol., 16: 1-18

Bradbury, P.J., Z. Zhang, D.E. Kroon, T.M. Casstevens, Y. Ramdoss and E.S. Buckler, 2007. TASSEL: software for association mapping of complex traits in diverse samples. Bioinformatics, 23: 2633-2635

Breseghello, F. and M.E. Sorrells, 2006a. Association analysis as a strategy for improvement of quantitative traits in plants. Crop Sci., 46: 13231330

Breseghello, F. and M.E. Sorrells, 2006b. Association mapping of kernel size and milling quality in wheat (Triticum aestivum L.) cultivars. Genetics, 172: 1165-1177

Cong, B., L.S. Barrero and S.D. Tanksley, 2008. Regulatory change in YABBY-like transcription factor led to evolution of extreme fruit size during tomato domestication. Nat. Genet., 40: 800-804

Dai, F., E. Nevo, D. Wu, J. Comadran, M. Zhou, L. Qiu, Z. Chen, A Beiles, G. Chen and G. Zhang, 2012. Tibet is one of the centers of domestication of cultivated barley. PNAS, 109: 16969-16973
Evanno, G., S. Regnaut and J. Goudet, 2005. Detecting the number of clusters of individuals using the software STRUCTURE: a simulation study. Mol. Ecol., 14: 2611-2620

Feng, Z.Y., L.L. Zhang, Y.Z. Zhang and H.Q. Ling, 2006a. Genetic diversity and geographical differentiation of cultivated six-rowed naked barley landraces from the Qinghai-Tibet plateau of China detected by SSR analysis. Genet. Mol. Biol., 29: 330-338

Feng, Z.Y., X.J. Liu, Y.Z. Zhang and H.Q. Ling, 2006b. Genetic diversity analysis of Tibetan wild barley using SSR markers. Acta. Genet. Sin. 33: 917-928

Flint-Garcia, S.A., J.M. Thornsberry and B.E. Th, 2003. Structure of linkage disequilibrium in plants. Annu. Rev. Plant. Biol., 54: 357-374

Gegas, V.C., A. Nazari, S. Griffiths, J. Simmonds, L. Fish, S. Orford, L. Sayers, J.H. Doonan and J.W. Snape, 2010. A genetic framework for grain size and shape variation in wheat. Plant Cell., 22: 1046-1056

Gupta, P.K., S. Rustgi and P.L. Kulwal, 2005. Linkage disequilibrium and association studies in higher plants: present status and future prospects. Plant Mol. Biol., 57: 461-485

Hadjichristodoulou, A., 1990. Stability of 1000-grain weight and its relation with other traits of barley in dry areas. Euphytica, 51: 11-17

Han, H.Q., Y. Liu, M.M. Jiang, H.Y. Ge and H.Y. Chen, 2015. Identification and expression analysis of YABBY family genes associated with fruit shape in tomato (Solanum lycopersicum L.). Genet. Mol. Res., 14: 7079-7091

Huang, B.G. and W.R. Wu, 2011. Mapping of mutant gene prbs controlling poly-row-and-branched spike in barley (Hordeum vulgare L.). $J$. Interg. Agric., 10: 1501-1505

Irfan, M., T.T. Zhang, Y. Wang, C. Zhang, Q. Miao, L. Zhang and F. Lin, 2013. Modification of CTAB protocol for maize genomic DNA extraction. Res. J. Biotechnol., 8: 41-45

Kjaer, B. and J. Jensen, 1996. Quantitative trait loci for grain yield and yield components in a cross between a six-rowed and a two-rowed barley. Euphytica, 90: 39-48

Korff, M.V., H. Wang, J. Léon and K. Pillen, 2004. Development of candidate introgression lines using an exotic barley accession (Hordeum vulgare ssp. spontaneum) as donor. Theor. Appl. Genet., 109: $1736-1745$

Korff, M.V., H. Wang, J. Léon and K. Pillen, 2006. AB-QTL analysis in spring barley: II. Detection of favourable exotic alleles for agronomic traits introgressed from wild barley ( $H$. vulgare ssp. spontaneum). Theor. Appl. Genet. 112: 1221-1231

Kulwal, P., G. Ishikawa, D. Benscher, Z. Feng, L.X. Yu, A. Jadhav, S. Mehetre and M.E. Sorrells, 2012. Association mapping for preharvest sprouting resistance in white winter wheat. Theor. Appl. Genet., 125: 793-805

Li, J.Z., X.Q. Huang, F. Heinrichs, M.W. Ganal and M.S. Röder, 2005. Analysis of QTLs for yield, yield components, and malting quality in a $\mathrm{BC}_{3}-\mathrm{DH}$ population of spring barley. Theor. Appl. Genet., 110: 356-363

Liu, K. and S.V. Muse, 2005. POWERMARKER: integrated analysis environment for genetic marker data. Bioinformatics, 21: 2128-2129

Locatelli, A., A. Cuestamarcos, L. Gutiérrez, P.M. Hayes, K.P. Smith and A.J. Castro, 2013. Genome-wide association mapping of agronomic traits in relevant barley germplasm in Uruguay. Mol. Breed., 31: $631-654$

Long, N.V., O. Dolstra, M. Malosetti, B. Kilian, A. Graner, R.G. Visser and C.G. Linden, 2013. Association mapping of salt tolerance in barley (Hordeum vulgare L.). Theor. Appl. Genet., 126: 2335-2351

Marquezcedillo, L.A., P.M. Hayes, A. Kleinhofs, W.G. Legge, B.G. Rossnagel, K. Sato, S.E. Ullrich and D.M. Wesenberg, 2001. QTL analysis of agronomic traits in barley based on the doubled haploid progeny of two elite North American varieties representing differen germplasm groups. Theor. Appl. Genet., 103: 625-637

Matthies, I.E., T.V. Hintum, S. Weise and M.S. Röder, 2012. Population structure revealed by different marker types (SSR or DArT) has an impact on the results of genome-wide association mapping in European barley cultivars. Mol. Breed., 30: 951-966

Maurer, A., V. Draba and K. Pillen, 2016. Genomic dissection of plant development and its impact on thousand grain weight in barley through nested association mapping. J. Exp. Bot., 67: 2507-2518 
Massman, J., B. Cooper, R. Horsley, S. Neate, R. Dill-Macky, S. Chao, Y. Dong, P. Schwarz, G.J. Muehlbauer and K.P. Smith, 2011. Genomewide association mapping of Fusarium head blight resistance in contemporary barley breeding germplasm. Mol. Breed., 27: 439-454

Mikolajczak, K., P. Ogrodowicz, K. Gudyś, K. Krystkowiak, A. Sawikowska, W. Frohmberg, A. Górny, A. Kedziora, J. Jankowiak, D. Józefczyk, G. Karg, J. Andrusiak, P. Krajewski, I. Szarejko, M. Surma, T. Adamski, J. Guzy-Wróbelska and A. Kuczyńska, 2016. Quantitative trait loci for yield and yield-related traits in spring barley populations derived from crosses between European and Syrian cultivars. Plos One, 11: 1-26

Passarella, V.S., R. Savin and G.A. Slafer, 2005. Breeding effects on sensitivity of barley grain weight and quality to events of high temperature during grain filling. Euphytica, 141: 41-48

Pillen, K., A.Zacharias and J. Léon, 2003. Advanced backcross QTL analysis in barley (Hordeum vulgare L.). Theor. Appl. Genet., 107: 340-352

Pritchard, J.K., M. Stephens and P. Donnelly, 2000. Inference of population structure using multilocus genotype data. Genetics, 155: 945-959

Rasheed, A., X. Xia, F. Ogbonnaya, T. Mahmood, Z. Zhang, A. MujeebKazi and Z. He, 2014. Genome-wide association for grain morphology in synthetic hexaploid wheats using digital imaging analysis. BMC Plant Biol., 14: 1-21

Riemann, M., C. Gutjahr, A. Korte, M. Riemann, B. Danger, T. Muramatsu, U. Bayer, F. Waller, M. Furuya and P. Nick, 2007. GER1, a GDSL motif-encoding gene from rice is a novel early light- and jasmonateinduced gene. Plant Biol., 9: 32-40

Royo, C., M. Abaza, R. Blanco and D.M.L.F. García, 2000. Triticale grain growth and morphometry as affected by drought stress, late sowing and simulated drought stress. Funct. Plant Biol., 27:1051-1059

Saal, B., M.V. Korff, J. Léon and K. Pillen, 2011. Advanced-backcross QTL analysis in spring barley: IV. Localization of QTL $\times$ nitrogen interaction effects for yield-related traits. Euphytica, 177: 223-239

Schnaithmann, F. and K. Pillen, 2013. Detection of exotic QTLs controlling nitrogen stress tolerance among wild barley introgression lines. Euphytica, 189: 67-88

Sun, L., D. Ma, H. Yu, F. Zhou, Y. Li, L. Luo, G. Gao, Q. Zhang, C. Xu and $\mathrm{Y}$. He, 2013. Identification of quantitative trait loci for grain size and the contributions of major grain-size QTLs to grain weight in rice. Mol. Breed., 31: 451-461

Szücs, P., V.C. Blake, P.R. Bhat, S. Chao, T.J. Close, A. Cuesta-Marcos, G.J. Muehlbauer, L. Ramsay, R. Waugh and P.M. Hayes, 2009. An integrated resource for barley linkage map and malting quality QTL alignment. Plant Genom., 2: 134-140

Tinker, N.A., D.E. Mather, B.G. Rossnagel, K.J. Kasha, A. Kleinhofs, P.M. Hayes, D.E. Falk, T. Ferguson, L.P. Shugar, W.G. Legge, R.B. Irvine, T.M. Choo, K.G. Briggs, S.E. Ullrich, J.D. Franckowiak, T.K. Blake, R.J. Graf, S.M. Dofing, M.A. Saghai Maroof, G.J. Scoles, D. Hoffman, L.S. Dahleen, A. Kilian, F. Chen, R.M. Biyashev, D.A. Kudrna and B.J. Steffenson, 1996. Regions of the genome that affect agronomic performance in two-row barley. Crop Sci., 36: 1053-1062

Varshney, R.K., T.C. Marcel, L. Ramsay, J. Russell, M.S. Röder, N. Stein, R. Waugh, P. Langridge, R.E. Niks and A. Graner, 2007. A high density barley microsatellite consensus map with 775 SSR loci Theor. Appl. Genet., 114: 1091-1103
Virender, S. and G.P. Zhang, 2003. Important quality attributes of malt barley (Hordeum vulgare L.): effect of genetics, environments and agronomic factors. J. Interg. Agric., 2: 815-824

Walker, C.K., R. Ford, M. Muñoz-Amatriaín and J.F. Panozzo, 2013. The detection of QTLs in barley associated with endosperm hardness, grain density, grain size and malting quality using rapid phenotyping tools. Theor. Appl. Genet., 126: 2533-2551

Wallwork, M.A.B, C.F. Jenner, S.J. Logue and M. Sedgley, 1998. Effect of high temperature during grain-filling on the structure of developing and malted barley grains. Ann. Bot., 82: 587-599

Wang, J., G. Sun, X. Ren, C. Li, L. Liu, Q. Wang, B. Du and D. Sun, 2016. QTL underlying some agronomic traits in barley detected by SNP markers. BMC Genet., 17: 1-13

Wenzel, A., T. Frank, G. Reichenberger, M. Herz and K.H. Engel, 2015. Impact of induced drought stress on the metabolite profiles of barely grain. Metabolomics, 11: 454-467

Worch, S., K. Rajesh, V.T. Harshavardhan, C. Pietsch, V. Korzun, L. Kuntze, A. Börner, U. Wobus, M.S. Röder and N. Sreenivasulu, 2011. Haplotyping, linkage mapping and expression analysis of barley genes regulated by terminal drought stress influencing seed quality. BMC Plant Biol., 11: 1-14

Xing, Y. and Q. Zhang, 2010. Genetic and molecular basis of rice yield. Annu. Rev. Plant Biol., 61: 421-442

Yan, L., A. Loukoianov, A. Blechl, G. Tranquilli, W. Ramakrishna, P. SanMiguel, J.L. Bennetzen, V. Echenique and J. Dubcovsky, 2004. The wheat VRN2 gene is a flowering repressor down-regulated by vernalization. Science, 303: 1640-1644

Yeh, F.C. and T. Boyle, 1999. POPGENE Version 1.32. The user-friendly software for population genetic analysis. University of Alberta and CIFOR, Calgary

Yu, J., G. Pressoir, W.H. Briggs, I.V. Bi, M. Yamasaki, J.F. Doebley, M.D. McMullen, B.S. Gaut, D.M. Nielsen, J.B. Holland, S. Kresovich and E.S. Buckler, 2006. A unified mixed-model method for association mapping that accounts for multiple levels of relatedness. Nat. Genet., 38: 203-208

Zhou, H., B.J. Steffenson, G. Muehlbauer, R. Wanyera, P. Njau and S. Ndeda, 2014. Association mapping of stem rust race TTKSK resistance in US barley breeding germplasm. Theor. Appl. Genet., 127: 1293-1304

Zhou, H. and B. Steffenson, 2013. Genome-wide association mapping reveals genetic architecture of durable spot blotch resistance in US barley breeding germplasm. Mol. Breed., 32: 139-154

Zhou, H., S. Liu, Y. Liu, Y. Liu, J. You, M. Deng, J. Ma, G. Chen, Y. Wei, C. Liu and Y. Zheng, 2016. Mapping and validation of major quantitative trait loci for kernel length in wild barley (Hordeum vulgare ssp. spontaneum). BMC Genet., 17: 1-9

Ziems, L.A., L.T. Hickey, C.H. Hunt, E.S. Mace, G.J. Platz, J.D. Franckowiak and D.R. Jordan, 2014. Association mapping of resistance to Puccinia hordei in Australian barley breeding germplasm. Theor. Appl. Genet., 127: 1199-1212

(Received 09 February 2017; Accepted 07 June 2017) 